\title{
Confocal Microscopy, Modeling and Quantitation of Corroded and Non-corroded Casts of Microvascular Systems.
}

\author{
R. C. Wagner*, K. Czymmek, ${ }^{*}$ and F. E. Hossler** \\ * Dept. of Biological Sciences, University of Delaware, Newark, Delaware 19716 USA \\ ** Dept. of Anatomy and Cell Biology, Box 70582, J. H. Quillen College of Medicine \\ East Tennessee State University, Johnson City, Tennessee 37614 USA
}

Conventional SEM of corrosion-casted tissues provide excellent views of the three-dimensional (3-D) surface morphology and distribution of blood vessel and capillary networks. Images of casts have remarkable depth and sharpness. However, when the specimen is tilted, the relationships and relative dimensions of the casted vessels change in a non-linear fashion due to parallax. It is difficult, therefore, to obtain quantitative data (1) and the interior organization of compact vessel systems such as the glomerulus of kidneys is not revealed.

Corrosion-casted microvascular systems can be viewed with confocal microscopy due to the intense fluorescent properties of blue Mercox (2). Surface renderings of z-series provide models of the interior of vessels that can be viewed and analyzed from any angle as 3-D data sets. Casted vessel systems can also be imaged uncorroded, as frozen sections stained with fluorescent dyes. Confocal microscopy of such specimens reveal not only the casting material filling the vessels but also the surrounding cell architecture and their relationship to the vessels (3)

We have casted kidney glomeruli and fish gill lamellae capillaries and obtained confocal z-series of both corrosion-casted and frozen sections of non-corroded Syto $13^{\mathrm{TM}}$ stained nuclei in specimens. Surface rendered models of corrosion-casted kidney glomeruli and fish gills provided realistic representations of the capillary interiors (Figs. 1\& 2) with the volume and surface area of the blood space readily computed. In addition, skeletonization of 3-D data sets permitted visualization of the interior organization of compact capillary systems (Fig. 3), which was not revealed in the rendered model. Skeletonization also enabled measurements of skeletal length, indicating the total length of the capillaries and branch points, as well as the degree of arborization (Fig. 4)

Confocal 3-D data sets of non-corroded specimens, exhibited not only the casted vessels but the nuclei and cytoplasm of cells surrounding the capillaries (Figs. 5 \& 6)). The casted vessels and uncorroded tissue cells can be individually visualized and surface modeled in separate channels.

In conclusion, the interior of blood vascular systems can be visualized in their entirety and 3-D parameters such as volume, surface area, length and branching can be easily quantified by computer analysis of corrosion cast confocal data sets.

(1) F. E. Hossler and J. E. Douglas, Micros. Microanal. 7 (2001) 253

(2) A. C. Nelson, Scanning Microsc 1 (1987) 817

(3) K. Czymmek, R. C. Wagner, F. E. Hossler, R. Kao, Micros. Microanal 6 (2001) 562 


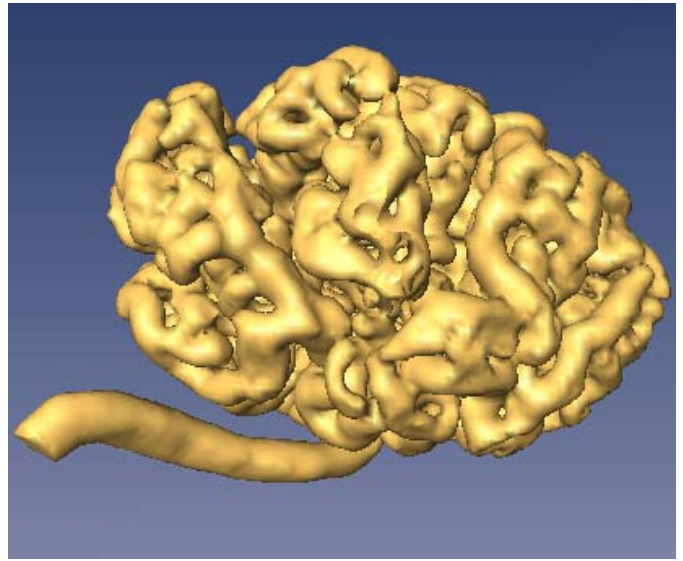

Figure 1. Surface-rendered model of a confocal z-series of a kidney glomerulus corrosion cast.

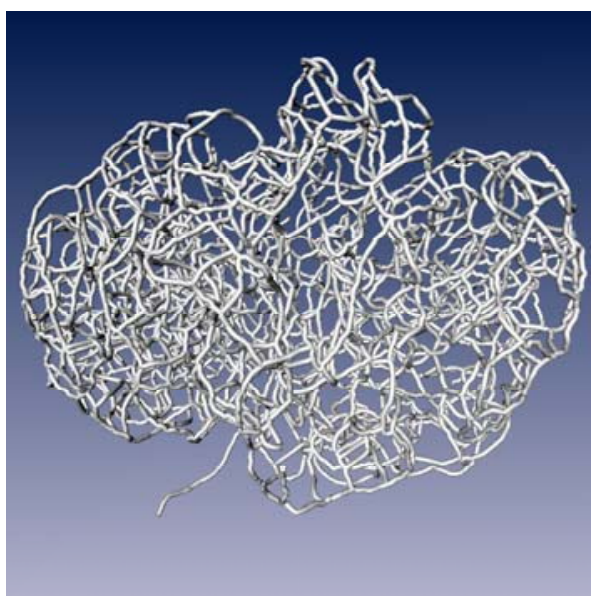

Figure 3. Skeletonized model of a 3-D data set of the kidney glomerulus shown in figure 1.

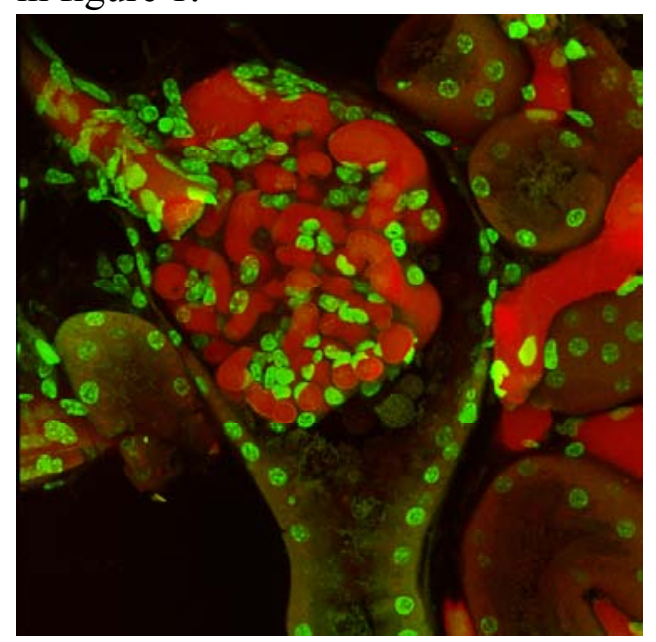

Figure 5. Confocal image showing casted glomerular capillaries (red) and surrounding nuclei of endothelial cells, podocytes and cells of Bowman's capsule.

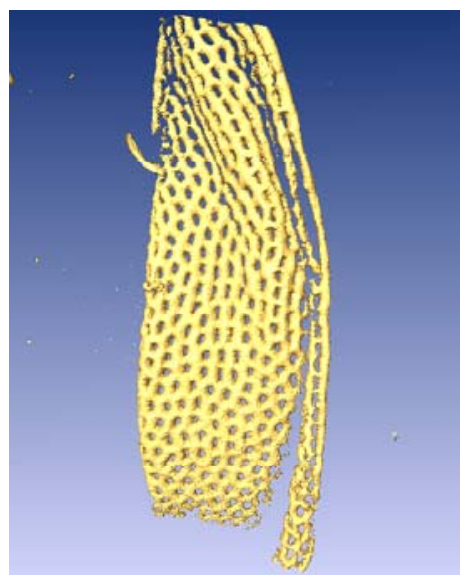

Figure 2. Surface-rendered model of a confocal z-series of corrosioncasted fish gill capillaries.

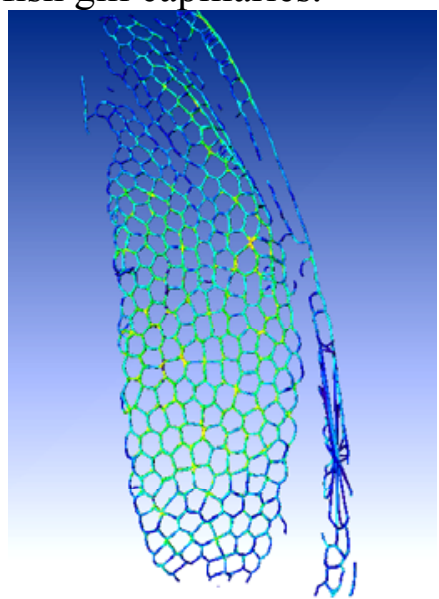

Figure 4. Skeletonized model of a 3-D data set of the fish gill shown in figure 2 .

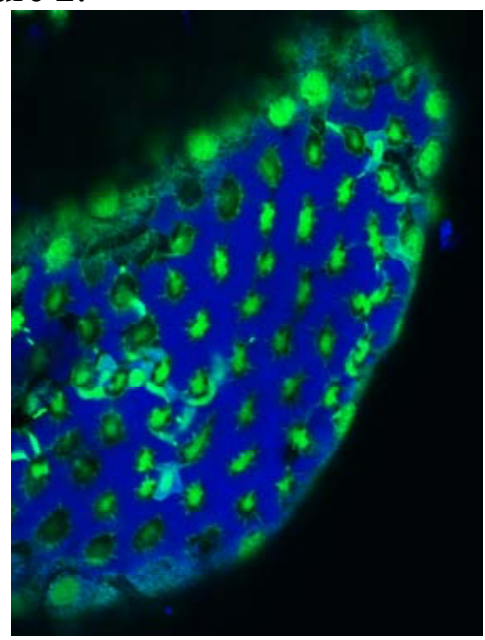

Figure 6. Confocal image showing casted capillaries (blue) and nuclei of surrounding cells (green). 